\title{
Féeries
}

Études sur le conte merveilleux, XVII $-\mathrm{XIX}{ }^{\mathrm{e}}$ siècle

\section{Les avatars de la "gaieté »: le dialogue du conte et de la fable chez La Fontaine}

The Avatars of "la Gaieté": the Dialogue of Tale and Fable in La Fontaine's Works

\section{Federico Corradi}

\section{(2) OpenEdition}

1 Journals

Édition électronique

URL : http://journals.openedition.org/feeries/757

DOI : $10.4000 /$ feeries.757

ISSN : 1957-7753

Éditeur

UGA Éditions/Université Grenoble Alpes

\section{Édition imprimée}

Date de publication : 31 octobre 2010

Pagination : 75-93

ISBN : 978-2-84310-182-3

ISSN : 1766-2842

\section{Référence électronique}

Federico Corradi, «Les avatars de la " gaieté » : le dialogue du conte et de la fable chez La Fontaine », Féeries [En ligne], 7 | 2010, mis en ligne le 31 juillet 2011, consulté le 10 décembre 2020. URL : http:// journals.openedition.org/feeries/757 ; DOI : https://doi.org/10.4000/feeries.757

\section{(c) Féeries}


Federico Corradi

Université Paris 3

\section{LES AVATARS DE LA "GAIETÉ" : LE DIALOGUE DU CONTE ET DE LA FABLE CHEZ LA FONTAINE}

$L$

E CONTE ET LA FABLE: deux genres dont les affinités et les entrecroisements paraissent évidents au XVII ${ }^{\mathrm{e}}$ siècle. Le Dictionnaire de Furetière a recours à la même terminologie pour les définir : "fiction", "chose inventée», "plaisanterie», "plaisant». Pour les contemporains de La Fontaine, les deux genres participent également de la «feinte» - la fable par statut, le conte par prédilection — et leur finalité avouée est celle de "plaire», de "divertir». Bien sûr, il existe aussi des contes "sérieux", c'est le cas des "histoires tragiques" mises à la mode par Bandello et par son émule Rosset, mais Furetière fait la part belle dans sa définition à la tradition de la «burla", représentée par les exemples de Noël du Fail, de Bonaventure de Périers et de Marguerite de Navarre. Seule différence substantielle, le rapport que les deux genres entretiennent avec l'instruction morale : pour la fable ce rapport est indispensable, pour le conte ce n'est qu'une virtualité. Prenons la définition de la Nouvelle:

Nouvelle, est aussi une histoire agreable \& intriguée, ou un conte plaisant un peu estendu, soit qu'elle soit feinte, ou veritable. Les nouvelles de Cervantes, de Scarron. Les cent nouvelles nouvelles, qu'on attribuë au Roi Louis XI. La nouvelle de Belphegor, la nouvelle Allegorique, \&c.

Bien que les termes utilisés soient pour la plupart les mêmes, on peut remarquer quelques différences. Si la définition du conte ne comporte pas d'indications quantitatives, la nouvelle doit être "un peu étendue» et «intriguée». Cette définition s'accorde mieux avec les nouvelles de Cervantès et de Scarron, qui occupent une place de choix parmi les exemples cités, qu'avec celles de Boccace, qui relèvent davantage de la tradition facétieuse et par conséquent sont axées plus rigoureusement sur une seule action simple et linéaire qui va droit à son but. On peut observer ici les premiers signes d'une spécialisation du mot "nouvelle» qui, à l'époque de la première édition du Dictionnaire, s'applique de préférence au récit à la manière des Espagnols, mais aussi à la nouvelle historique 
de Madame de La Fayette et de Saint-Réal et à la nouvelle galante de Madame de Villedieu. De même, chez La Fontaine, les deux termes apparaissent d'abord comme synonymes ${ }^{\mathrm{I}}$ - les trois premières parutions de I664, I665 et I666 s'intitulent Nouvelles en vers tirée [sic] de Boccace et de l'Arioste par M. de L. F., Contes et Nouvelles en vers de M. de La Fontaine et Deuxième Partie des Contes et Nouvelles en vers - mais le mot "conte" s'impose avec les Nouveaux Contes de Monsieur de La Fontaine (I674). La Fontaine semble donc prendre progressivement conscience d'aller au rebours de l'évolution la plus récente du genre : dès i656, les devisantes du récit-cadre des Nouvelles françaises de Segrais avaient proposé de chercher des aventures "extrêmement naturelles, tendres et surprenantes", qui auraient pour protagonistes "des chevaliers ou des princes français» et qui seraient situées dans un passé récent. En 1662, La Princesse de Montpensier réalise les vœux de Segrais par un récit solidement centré sur une intrigue amoureuse, soucieux de la vraisemblance narrative et situé dans un cadre historique que la connaissance des historiens et des mémorialistes (notamment Davila et Mézeray) permet de dessiner avec précision : l'exemple sera suivi au début des années I670 par Madame de Villedieu dans ses Annales galantes et par Saint-Réal dans Don Carlos. La valeur exemplaire de ces récits, affichant en général une conception sérieuse et désabusée de la passion amoureuse, est accrue par le choix de personnages appartenant aux familles les plus illustres du royaume. Négligeant complètement cette évolution, La Fontaine choisit de rester fidèle à Boccace, tout en opérant une notable restriction thématique : il ne retient de son modèle que les canevas licencieux et il met le genre sous le signe de la fiction avouée et de l'évasion ludique, en un mot de la "gaieté». Or la gaieté, clef de voûte de l'écriture des Contes, deviendra également un mot-clé de la poétique des Fables. La Fontaine opère ainsi un sensible rapprochement des deux genres, encore plus marqué que dans la tradition précédente (il faut rappeler que les recueils de "facéties" dès le $\mathrm{XVI}^{\mathrm{e}}$ siècle faisaient souvent place à des apologues, ce qui rend cette affinité plus évidente ${ }^{2}$ ). Il en fait deux manifestations différentes d'une même veine, d'une même modalité nar-

I. Sauf si on veut reconnaitre, comme le fait J.-P. Collinet, une différence entre les «nouvelles» plus étendues en vers irréguliers tirées de l'Arioste et les "contes» en octosyllabes et en vieux langage qui, pour la plupart, tirent leurs sujets de Boccace. Mais rien dans l'Avertissement de 1664 ne semble justifier cette répartition terminologique.

2. Pour le rapport de La Fontaine avec la tradition facétieuse, voir G. Peureux, «Arétin mitigé ou Boccace des ruelles? Les ascendances facétieuses des Contes dans l'Italie de la Renaissance", Le Fablier, ${ }^{\circ}{ }^{\circ} 4,2002$, p. 49-54. 
rative ${ }^{3}$, qu'on peut définir "poétique de la gaieté» : à mon sens elle consiste notamment à souligner à loisir, par des interventions ironiques de la voix d'auteur, le caractère artificiel et fictionnel de la narration. Cette ironie métalinguistique permet à La Fontaine de «faire passer» des «bagatelles» qui autrement ne seraient pas reçues par le public exigeant et raffiné auquel il s'adresse : le lecteur peut ainsi accéder à des sources de plaisir interdites, que ce soit le plaisir voyeuriste du conte grivois ou le plaisir régressif de la fable animalière, essentiellement liée avant La Fontaine à l'enseignement des collèges ou à la délectation érudite de quelques humanistes. Cela n'amène pas La Fontaine à effacer les différences entre les Fables et les Contes (d'ailleurs il a continué à mener de front ces deux modes d'écriture sans que l'un s'impose jamais définitivement sur l'autre) : mon propos est précisément celui de définir les modalités et les enjeux de la "poétique de la gaieté », compte tenu des exigences différentes auxquelles elle répond dans les deux genres. Nous essayerons de dégager les principes de cette poétique à partir du complexe appareil paratextuel qui accompagne les premiers recueils (pour les Contes, l'Avertissement de la plaquette de I664 et les deux préfaces de I665 et I666, pour les Fables l'épître en prose à Monseigneur le Dauphin et la Préface du recueil de I668), avant de les tester par l'analyse comparée d'un conte et d'une fable.

\section{La mise à distance des modèles}

On peut d'abord observer que, dans ses préfaces, La Fontaine adopte une attitude qu'on pourrait définir comme archéologique : il dédaigne complètement la tradition la plus récente des deux genres pour se rattacher directement aux pères fondateurs, aux divinités tutélaires de ces deux traditions, qui se situent à une énorme distance par rapport à lui. Ce retour aux sources mêmes du genre lui permet de se ménager un espace d'autonomie par rapport aux autorités de son siècle et aux jugements de la critique. Dans la préface des Fables de i668, les autorités de Phèdre et

3. Les contemporains reconnaissaient implicitement l'unité d'inspirations des Contes et des Fables, apparentés par une certaine modalité d'écriture: qu'on se souvienne du jugement de Madame de Sévigné, qui blâme La Fontaine pour avoir voulu s'écarter de son talent de conteur à l'occasion de la parution, en I67I, du volume mêlé de Fables nouvelles et autres poésies : "Je voudrais faire une fable qui lui fit entendre combien cela est misérable de forcer son esprit à sortir de son genre, et combien la folie de vouloir chanter sur tous les tons fait une mauvaise musique. Il ne faut point qu'il sorte du talent qu'il a de conter.» (Correspondance, I, R. Duchêne (éd.), Paris, Gallimard, coll. «Bibliothèque de la Pléiade», I972, p. 247.) 
de Socrate lui servent d'abri contre le verdict de Patru interdisant aux fabulistes l'usage du vers ${ }^{4}$. De même, dans les Contes (Préface, I665), Horace et l'Arioste (considéré à cette époque déjà comme un classique) sont allégués successivement pour rejeter le reproche d'immoralité que les premiers contes s'étaient attirés. Cela n'empêche pas que La Fontaine ne se démarque aussi, plus subtilement, des autorités anciennes : l'impossibilité alléguée d'imiter l' "élégance " et "l'extrême brèveté" du latin de Phèdre lui donne le droit d' "égayer l'ouvrage plus qu'il n'a fait ${ }^{6}$ ». L'appel au "goût du siècle", dans sa modestie apparente, a justement la fonction d'assurer l'autonomie de l'imitateur moderne. Dans les Contes (Préface, I666), qui ne sont que "bagatelles", relevant d'un genre moderne et non codifié, La Fontaine n'a plus besoin de tant de détours pour affirmer son indépendance : si Térence, entre autres, a contaminé et transformé les sujets qu'il a tirés de Ménandre, "ce privilège cessera-t-il à l'égard des contes faits à plaisir ${ }^{7}$ ?». Cet argument "d'autorité» suffit pour justifier «la liberté que l'auteur se donne de tailler dans le bien d'autrui ainsi que dans le sien propre, sans qu'il en excepte les nouvelles même les plus connues, ne s'en trouvant point d'inviolable pour $\mathrm{lui}^{8}{ }^{\circledR}$. Cette attitude d'indépendance habilement justifiée dans le paratexte permet de s'écarter de la route tracée par ses prédécesseurs et d'opérer un rapprochement sans précédents des deux genres, unifiés par une manière, par des procédés de style qui sont bien de La Fontaine et qu'il désigne par le mot de "gaieté».

En effet, les Contes et les Fables représentent pour La Fontaine deux champs parallèles d'expérimentation qui s'ouvrent à différentes possibilités de développement (dans l'Avertissement de i664, il fait appel au jugement du public pour décider du choix entre deux manières ou deux "caractères» différents : en effet, une nouvelle plus développée en vers irréguliers, Joconde, côtoie dans la plaquette un récit plus resserré en octosyllabes, Le Cocu, battu et content). Le résultat dans les deux cas est un renouvellement radical, qui se résume symboliquement dans le choix du vers. Ce choix, qui n'allait de soi ni pour les Contes ni pour les Fables, permet de resserrer les liens entre les deux genres qui, dans leur configuration traditionnelle, étaient sans doute moins proches. C'est par le vers que la "poétique de la gaieté» peut le mieux se déployer. Dans les deux cas, la

4. J. de La Fontaine, Euvres complètes, t. I, Fables et Contes (= EC ), éd. établie, présentée et annotée par J.-P. Collinet, Paris, Gallimard, coll. «Bibliothèque de la Pléiade», I991, p. 5-6.

5. Ibid., p. 556.

6. Ibid., p. 7.

7. Ibid., p. 605 .

8. Ibid., p. 604 . 
mise en vers est revendiquée comme le mérite principal, le seul peut-être, de l'entreprise du poète : c'est à ce propos qu'il dit dans la préface des Contes " [s'être] engagé dans une carrière toute nouvelle"». Parallèlement, pour les Fables, il se flatte que faute d'avoir couru dans cette carrière avec succès "[on lui donnera] au moins la gloire de l'avoir ouverte ${ }^{10}$ ». Mais il y a d'autres éléments qui reviennent, d'autres notions qui rapprochent les deux «carrières» du poète. Patrick Dandrey a déjà fait remarquer que le lexique employé par La Fontaine pour caractériser les deux genres est presque indifférencié, ce qui n'implique nullement que les différences soient en effet effacées ${ }^{\text {II }}$.

\section{La poétique du conte et de la fable : affinités et différences}

D'abord la "gaieté» : cette notion est utilisée dans deux passages-clés, toujours à la conclusion d'un raisonnement où La Fontaine justifie ses hardiesses par rapport à la tradition. Dans la première préface des Contes (I665), il s'agit de rejeter une conception trop étroite de la bienséance, qui «[réduirait] Boccace à la même pudeur que Virgile ${ }^{12}$ ». Cela amène La Fontaine à établir la spécificité du conte vis-à-vis du roman : c'est une différence tonale qui marque la frontière des deux genres, "gaieté» d'une part, «douce mélancolie» de l'autre. Mais on peut considérer que la même frontière sépare La Fontaine de la nouvelle à la manière de Madame de La Fayette qui, obéissant à un critère de vraisemblance, fournit un tableau saisissant des passions et fait appel à l'identification du lecteur, pour qu'il puisse se purger des "désordres» qu'elles peuvent amener. La nouvelle de modèle espagnol, à l'imitation de Cervantes, d'ailleurs n'en affiche pas moins un critère de vraisemblance ou même de vérité alléguée (c'est le cas de Sorel). La Fontaine va exactement dans le sens contraire : il «déréalise» la nouvelle, récusant nettement la pertinence des principes de vraisemblance et de vérité. "Ce n'est ni le vrai ni le vraisemblable qui font la beauté et la grâce de ces choses-ci; c'est seulement la manière de les

9. Ibid.

Io. Ibid., p. 6.

II. La Fabrique des Fables, Paris, Klincksieck, 1992, p. 37 et suiv. P. Dandrey ajoute d'ailleurs que ce rapprochement opéré entre un genre à vocation moralisante et un genre immoral relève du paradoxe et presque de la provocation. Tout mon développement est largement redevable de son analyse de la préface des Fables.

12. $E C$, p. 557. 
conter ${ }^{13}$.» L'utilité morale n'étant évidemment pas en jeu dans des contes licencieux, le seul critère qui reste pour décréter la réussite du conte est le "plaisir» du lecteur, déterminé par la "manière de conter». La clef de l'identité du genre est repérée dans une qualité de style, ou pour mieux dire dans la conformité entre les deux plans du style et du contenu (c'est ce que La Fontaine appelle «bienséance» en opérant un glissement adroit de l'acception morale à une acception purement esthétique du terme). Comment faut-il entendre cette conformité? La matière licencieuse des contes, présentée comme principe non négociable du genre, doit se refléter dans les choix stylistiques, ce qui ne revient pas à employer des termes vulgaires, mais à refuser de parer le désir du langage mystifiant du sentiment. La gaieté n'est que ceci au fond : traiter avec le détachement convenable le désir sexuel sans les mystifications de l'idéalisme amoureux et romanesque ${ }^{\mathrm{I4}}$. "Gaieté» renvoie à «jeu ${ }^{15}$ »; les deux termes ont une double acception, métalittéraire et sexuelle : ils se réferent d'une part à une littérature finalisée au plaisir et destinée à la consommation immédiate, d'autre part à une pratique de la sexualité dégagée de toute implication sentimentale qui guérit des effets de l'humeur mélancolique («Il est un jeu divertissant sur tous $\left.[\ldots]^{16} »\right)$.

Dans la Préface des Fables de 1668, La Fontaine, lorsqu'il définit les traits marquants du genre, le rapproche des Contes de manière assez inattendue. Notamment l'exigence de "brèveté», qui empêche "le conte» de "languir ${ }^{17}$ ». Cette exigence avait été déjà soulignée dans la préface des Contes de i666. C'est le point de départ qui est différent, parce que dans les Contes il s'agissait de "retrancher" plutôt que d' "enchérir ${ }^{18}{ }^{18}$ ", dans les Fables au contraire l'auteur se trouve dans la nécessité d'ajouter des ornements pour concilier les "grâces lacédémoniennes» avec les «Muses françaises ${ }^{19}$ ». D'ailleurs, de même que les Contes étaient des "bagatelles», les Fables sont des «badineries» dont l' "apparence [...] est puérile», ce qui rapproche d'emblée les deux genres par une certaine apparence de naï-

I3. Ibid.

I4. On retrouve au seuil de la nouvelle programmatique Les Oies de frère Philippe cette antithèse entre une représentation explicite et enjouée du désir et la représentation sublimée qu'en débitent les soupirants dans leurs "fleurettes»: «Irait-il après tout s'alarmer sans raison / pour un peu de plaisanterie? / Je craindrais bien plutôt que la cajolerie / ne mît le feu dans la maison. " (Ibid., p. 7 OI.)

15. "Qui ne voit que ceci est jeu et par conséquent ne peut porter coup?» (Ibid., p. 557.)

I6. Comment l'esprit vient aux filles, ibid., p. 8II.

17. Ibid., p. 5 .

I8. Ibid., p. 605.

19. Ibid., p. 5 . 
veté et par leur indifférence affichée pour le critère de la vraisemblance. Mais ce qui les apparente surtout, c'est encore une fois la poétique de la "gaieté». La Fontaine fait appel à cette notion pour justifier son choix d' "égayer" son ouvrage plus que ses modèles latins ne l'avaient fait, d'y ajouter «quelques traits qui en relevassent le goût ${ }^{20} »$. C'est à la conclusion de ce raisonnement que s’inscrit cette célèbre définition : "Je n’appelle pas gaieté ce qui excite le rire; mais un certain charme, un air agréable qu'on peut donner à toutes sortes de sujets, même les plus sérieux ${ }^{2 \mathrm{I}}$."

Faut-il en conclure que les deux genres n'en font plus qu'un? Non, de toute évidence. Seulement, La Fontaine nous invite à ne pas rechercher cette différence au niveau de la forme, où toute distinction semble abolie, mais à un niveau plus profond : les ambitions respectives des deux genres. Non pas tant la présence ou l'absence de la moralité explicite (d'ailleurs plusieurs Contes affichent une structure homologue à celle des Fables, avec une «moralité» qui précède ou suit le récit, et les Fables peuvent très bien se passer d'expliciter leur signification, quand celle-ci est aisée à suppléer), plutôt leur rapport avec la longue durée de la tradition poétique : les Contes ne prétendent pas à la longue durée, ils se posent d'emblée sous l'égide de la mode, de la variation, de la diversité. Cela explique la présence dans la préface des noms de Marot et de Voiture, modèles d'une poésie destinée à la consommation immédiate, d'une gaieté qui "passe légèrement» : "Il n'appartient qu'aux ouvrages vraiment solides, et d'une souveraine beauté, d'être bien reçus de tous les esprits, et dans tous les siècles, sans avoir d'autre passeport que le seul mérite dont ils sont pleins» et La Fontaine reconnaît que les siens «sont fort éloignés d'un si haut degré de perfection ${ }^{22}$ ». Cette solidité ne fait pas défaut aux Fables: "Ces badineries ne sont telles qu'en apparence; car dans le fond elles portent un sens très solide ${ }^{23}$." Du coup, les Fables, ce "livre favori" par lequel l'auteur "ose espérer une seconde vie ${ }^{24}$ », manifestent leur aspiration à s'inscrire dans la longue durée de la tradition poétique. Le recours aux topoï de la célébration humaniste de la poésie le confirme : la mise en vers n'est plus seulement un artifice technique visant à diversifier le plaisir, c'est une annexion au royaume de la Poésie. Par la référence à Socrate, à Platon et aux paraboles de l'Écriture, La Fontaine conserve l'ambiguïté

20. Ibid., p. 7 .

2I. Ibid.

22. Ibid., p. 555-556.

23. Ibid., p. 8.

24. À $M^{\text {me }}$ de Montespan, ibid., p. 248. 
de ses prédécesseurs entre fable animalière et Fable au sens large, le point de contact entre les deux étant le détour par la fiction pour accéder à la vérité. La fable est donc en rapport organique avec la vérité : elle se configure comme un effort herméneutique qui sous le voile de l'image essaie de porter un regard lucide sur l'univers des hommes.

Néanmoins, ces topoï n'étaient en rien nouveaux : ils étaient parfaitement connus du public et faisaient partie du discours traditionnel sur la fable. La Fontaine n'y ajoute rien, ce qui prouve que son attention est orientée ailleurs. L'originalité de cette préface réside plutôt dans l'attention réservée à la définition du style de l'apologue poétisé. L'appel à la «nouveauté» et au "goût du siècle» n'est pas innocent dans un ouvrage de ce genre, où la souveraineté de la mode est beaucoup moins légitime que dans les Contes: c'est sans doute une allusion discrète à son expérience de conteur, qui du coup se trouve inscrite à l'intérieur même de l'acte fondateur de l'apologue. Dépouillé de son acception sexuelle, le terme "gaieté» désigne pourtant encore une certaine qualité de style, une certaine gestion de la narration qui implique, comme dans les Contes, une adaptation du langage à la matière. Malgré leur rapport avec la Vérité, les fables comportent une réduction des vérités morales à la portée du public d'enfants auquel le fabuliste feint de s'adresser en priorité. La chair dont elles enveloppent leurs moralités (elles aussi souvent naïves et pléonastiques par rapport au récit qu'elles sont censées éclairer) est celle de la fiction la plus grossière et la plus enfantine : attribuer aux animaux langage et comportements humains. Peut-on traiter de semblables sornettes autrement que par un ton d'ironie et de détachement qui apparente l'écriture à celle des Contes? Le point de contact essentiel entre les deux genres ne peut être donc qu'une certaine modalité narrative qui comporte une présence discrète de la voix d'auteur, teintée d'ironie légère et de supériorité amusée par rapport aux personnages mis en scène. Dans le cas des Contes, ce qu'il faut tenir à distance, c'est le potentiel transgressif et antisocial de la pulsion sexuelle dépouillée de tout voile mystifiant, dans le cas des Fables c'est le désir régressif que recèle une fiction enfantine destinée en réalité à un public adulte. Paradoxe d'une régression consentie mais contrôlée qui se dessine dans la célèbre conclusion du Pouvoir des fables : "Le monde est vieux, dit-on; je le crois, cependant / Il le faut amuser encore comme un enfant ${ }^{25}$." Certes, La Fontaine applique cette expression à un conte de vieille comme Peau d'Âne, dépourvu avant Perrault de toute signification

25. Ibid., p. 297. 
morale qui le rachète de sa puérilité, mais il l'annexe à la plus large catégorie des "contes d'enfants", dont il illustre par l'exemple de l'orateur à la fois le pouvoir et les dangers. Réjouir le public par des fables d'animaux, c'est flatter les instincts les plus bas de l'auditoire, ceux mêmes qui l'empêchent de prêter l'oreille à l'éloquence sublime et inspirée de l'orateur, mais c'est aussi recourir au moyen le plus sûr pour le captiver. Si l'orateur de la fable semble mépriser le moyen dont il se sert pour conquérir l'attention des Athéniens, La Fontaine, reprenant la parole à la fin de son récit, atténue la polarité entre les intérêts sérieux de l'âge adulte et la puérilité des récits d'animaux : les "contes d'enfants", notamment les fables ésopiques dans la version moderne que La Fontaine en propose, sont une manière de ménager à la fois les plaisirs de l'enfance et la lucidité de la réflexion morale, ils font accéder le public à des plaisirs défendus tout en rachetant la gratuité de ces plaisirs par la complexité d'une pensée qui hérite de la réflexion et du regard lucide des moralistes.

\section{Les avatars de la gaieté}

Un verbe-clé qui apparaît dans les passages où La Fontaine définit la poétique des deux genres est "faire passer» (ou un synonyme comme "insinuer»). Dans les deux genres il y a quelque chose qui couvre et qui cache autre chose, en lui permettant de s'insinuer dans l'âme du lecteur. On sait que dans les fables "le conte fait passer le précepte avec lui ${ }^{26}$ »; mais il ne s'agit plus seulement de faire accepter une vérité morale grâce au plaisir procuré par le récit. Comme ces récits sont "[sus] de tout le monde ${ }^{27}$ ", ce sont eux qui ne seraient pas reçus sans le charme de la nouveauté et de la gaietée ${ }^{28}$. D'ailleurs, leur naïveté affichée rebuterait le rationalisme "cartésien» des hommes du XVII ${ }^{\mathrm{e}}$ siècle sans la caution d'une écriture qui souligne plaisamment cette invraisemblance par les jeux du langage et par la superposition plaisante et décalée des traits humains aux silhouettes animales. C'est donc le récit qu'il faut «faire passer" à l'aide de la nouveauté ingénieuse du style. Dans le cas des Contes, on peut recourir à un texte paradigmatique tel que Le Tableau: "Qui pense finement et s'exprime avec grâce fait tout passer, car tout passe ${ }^{29}$." Il s'agit donc de faire passer

26. Le Pâtre et le Lion, ibid., p. 209.

27. Ibid., p. 7.

28. Voir P. Dandrey, La Fabrique des Fables, ouvr. cité, p. 17.

29. Ibid., p. 887. 
un contenu grivois sans choquer les bienséances et sans recourir au voile de l'idéalisme romanesque. Il s'agit de mettre à distance la sexualité par le style, d'en atténuer la violence par la "gaieté», présentée à la fois comme un remède contre la mélancolie et la violence aveugle du désir ${ }^{30}$. De la gaieté d'ailleurs on dit qu'elle "passe légèrement ${ }^{3 \mathrm{I}}$ » : car cette sorte d'euphorie, proche du désir sexuel, est à la fois provoquée et assouvie par le voyeurisme du conte et ne se prolonge pas au-delà.

Si le but de La Fontaine dans les deux genres est de mettre à distance la matière, on comprend dès lors le caractère si ouvertement "réflexif» de sa production lyrico-narrative : toute l'attention passe de la «matière» à «la manière de conter». On demande au lecteur une réception raisonnée, complexe, où le plaisir régressif ou transgressif de la narration est justifié, "protégé» par les clins d'œil métalittéraires du narrateur, qui soulignent à loisir l'invraisemblance. C'est ce qu'a très bien exprimé l'auteur anonyme de la Dissertation sur Joconde, identifié avec Boileau :

Le secret donc en contant une chose absurde, est de s'énoncer d'une telle manière, que vous fassiés concevoir au Lecteur que vous ne croiés pas vous même la chose que vous lui contés. Car alors il aide lui même à se decevoir et ne songe qu'à rire de la plaisanterie agréable d'un Auteur qui se joüe et ne lui parle pas tout de bon ${ }^{32}$.

C’était déjà le sens du mot «badinerie» dans la préface de Psyché :

Dans un conte comme celui-ci, qui est plein de merveilleux à la vérité, mais d'un merveilleux accompagné de badineries, et propre à amuser des enfants, il a fallu badiner depuis le commencement jusqu’à la fin, il a fallu chercher du galant et de la plaisanterie ${ }^{33}$.

Les mots «badinerie» et "badiner» me semblent ici désigner à la fois ce qu'il y a d'invraisemblable et d'enfantin dans le conte de Psyché tel que la tradition le lègue à La Fontaine (une sorte de conte de fées "propre à amuser des enfants») et les procédés discursifs que La Fontaine met en

30. D'ailleurs cet usage "thérapeutique» de la gaieté comme antidote à la mélancolie se rattache très directement à l'image idéale du poète que Pellisson esquisse en faisant le portrait de Voiture, présenté comme celui qui a ramené la poésie française à l'esprit "gai et folâtre" de Marot et de Saint-Gelais : "Monsieur de Voiture [...] vint alors avec un esprit très galant et très délicat et une mélancolie douce et ingénieuse, de celles qui cherchent sans cesse à s'égayer. " (P. Pellisson, "Discours sur les œuvres de Monsieur Sarasin", dans A. Viala et al., L'Esthétique galante. Paul Pellisson. Discours sur les Euvres de Monsieur Sarasin et autres textes, Toulouse, Société de littératures classiques, 1989, p. 69.)

3I. $E C$, p. 557.

32. N. Boileau, Euvres complètes, introduction par A. Adam. Textes établis et annotés par F. Escal, Paris, Gallimard, coll. «Bibliothèque de la Pléiade», I996, p. 312.

33. J. de La Fontaine, Euvres complètes, t. II, Euvres diverses (= EDD), éd. établie et annotée par P. Clarac, Paris, Gallimard, coll. «Bibliothèque de la Pléiade», I958, p. I23-I24. 
œuvre pour souligner de manière plaisante cette invraisemblance («il a fallu badiner depuis le commencement jusqu’à la fin»). Ces procédés discursifs se retrouvent dans la "gaieté» des Contes et des Fables, qui consiste à instaurer par des jeux linguistiques une duplicité de sens qui demande une conscience en éveil ${ }^{34}$. C'est cette réflexivité qui permet de renouveler, de «faire passer» un matériau narratif qui n'est jamais original, mais au contraire "commun, usé et rebattu ${ }^{35}$ ", ce qui éloigne encore davantage La Fontaine de la tradition de la nouvelle, interprétée souvent par les auteurs et les théoriciens du genre comme une histoire nouvellement arrivée qu'on livre au public. La Fontaine, au contraire, affiche à loisir cette saturation culturelle par les allusions plaisantes à ses sources qu'il intègre au récit. La gaieté oblige le lecteur à focaliser l'attention sur au moins deux niveaux du texte à la fois : pour les Fables le monde animal et le monde humain qui se superposent plaisamment dans le récit, pour les Contes la réalité transgressive du désir sexuel et les langages sectoriels mobilisés pour la travestir (un de ces voiles "de gaze» qui ne cachent rien). Mais il faut compter aussi les textes avec lesquels s'esquisse un dialogue : "c'est une littérature qui s'ouvre sur une enfilade de littératures ${ }^{36}$ », c'est le mot de Jean Rousset sur Psyché qu'on pourrait appliquer à toute l'œuvre de La Fontaine. C'est donc essentiellement sur le plan de l'elocutio qu'on mesure l'originalité de La Fontaine par rapport à ses sources.

\section{La voix du conteur : La Fiancée du roi de Garbe}

Prenons deux exemples pour montrer la manière dont cette "poétique de la gaieté» se manifeste concrètement dans les deux genres. Dans La Fiancée du roi de Garbe l'application de ce "programme» est particulièrement évidente : La Fontaine pose d'emblée la question du rapport avec sa source avouée, Boccace. Le lecteur est invité explicitement à lire la nouvelle sans

34. C'est ce qu’a très bien remarqué A.-M. Bassy dans sa préface des Contes et Nouvelles en vers, Paris, Gallimard, coll "Folio», I982, p. I6 : La Fontaine inverse les règles du genre de la nouvelle, d'une littérature de l'affectus il fait une littérature de l'intellectus. "Il substitue les jeux de l'esprit et $d u$ sens aux jeux de l'émotion et des sens. [...] Entre la réalité du récit — c'est-à-dire de la "nouvelle" - et l'auditeur ou le lecteur, le "conte", chez La Fontaine, introduit l'épaisseur du langage ou de l'écriture."

35. La Matrone d'Éphèse, CEC, p. 508.

36. J. Rousset, "Psyché ou le plaisir des larmes», dans L'intérieur et l'extérieur. Essai sur la poésie et sur le théâtre au XVII siècle, Paris, Corti, 1968, p. II5. 
oublier le modèle. Toute réception naïve qui s'attacherait uniquement au plaisir «pulsionnel» du conte grivois est discréditée :

J'ai suivi mon auteur en deux points seulement :

Points qui font véritablement

Le plus important de l'histoire.

L'un est que par huit mains Alaciel passa

Avant que d'entrer dans la bonne :

L'autre que son fiancé ne s'en embarrassa, $[\ldots]^{37}$.

Prologue ouvertement métalittéraire, où La Fontaine démonte à l'intention des lecteurs le mécanisme de la nouvelle pour en mettre à nu les ressorts : c'est un pacte de lecture qu'il propose à son lecteur, un programme que lui-même s'engage à respecter. Cela revient à désamorcer le plaisir immédiat de la lecture au profit d'une appréciation plus subtile de la virtuosité narrative et des jeux intertextuels. Il ne faut pas oublier que la dimension parodique était déjà un enjeu essentiel de la nouvelle de Boccace : comme l'a remarqué Cesare Segre, le conteur italien adopte sciemment le schéma typique du roman grec (promesse de mariage - obstacles qui en retardent l'accomplissement - réalisation du mariage), mais il l'inverse en son essence : car les obstacles, au lieu de comporter la défense héroïque de la fidélité à l'époux promis, se terminent infailliblement par la capitulation de l'héroïne ${ }^{38}$. Le plaisir comique que le lecteur tire de la nouvelle gît dans le mécanisme répétitif qui oriente bientôt son attente vers la conclusion érotique de chaque séquence narrative, et dans la fraude finale qui préserve la possibilité du mariage par un jeu de mots burlesque qui annule la séquence des rapports sexuels intervenus au cours des pérégrinations de la protagoniste. Mais tandis que chez Boccace la parodie était confinée à la dispositio, le ton de la narration gardant toujours une impassibilité plus propre à souligner la violence d'un monde où la sexualité est prise dans un jeu de rapports de force, La Fontaine englobe l'elocutio dans le jeu parodique. C'est bien sur ce plan-là qu'il renchérit sur son modèle, contredisant par sa loquacité débordante l'adhésion alléguée à un idéal de brièveté et de continuité narrative ${ }^{39}$. Le modèle romanesque est pris à partie explicitement aussi bien pour ses entorses à la vraisemblance que pour cet idéalisme amoureux censé induire chez les jeunes personnes une "douce mélancolie» très dangereuse pour leur vertu. On peut citer

37. Ibid., p. 667.

38. Voir C. Segre, "Comicità strutturale nella novella di Alatiel», dans Le strutture e il tempo, Torino, Einaudi, 1974, p. I45-179.

39. Dans la préface de $1666, C E$, p. 605. 
le passage où le défaut de vraisemblance d'un certain artifice narratif est souligné avec une sorte de coquetterie au lieu d'être caché. Rien de tel chez Boccace, évidemment :

Pourquoi me dira-t-on, nous ramener toujours

Cette cassette? est-ce une circonstance

Qui soit de si grande importance?

Oui, selon mon avis; on va voir si j'ai tort.

Je ne prends point ici l'essor,

Ni n'affecte de railleries.

Si j'avais mis nos gens à bord

Sans argent et sans pierreries,

Seraient-ils pas demeurés court?

On ne vit ni d'air ni d'amour.

Les amants ont beau dire et faire,

Il en faut revenir toujours au nécessaire.

La cassette y pourvut avec maint diamant

$[\ldots]$

Voyez combien voilà de choses enchaînées,

Et par la cassette amenées ${ }^{40}$.

Pour la parodie de l'amour romanesque, qu'on se souvienne des amours d'Hispal et d'Alaciel, où le rituel de la séduction, qui dans les romans était la matière même de la narration et se prolongeait indéfiniment, est abrégé au maximum par le recours à l'infinitif de narration, comme si le narrateur voulait simplement adapter ce topos romanesque au temps diégétique beaucoup plus resserré du conte :

Pleurs de couler, soupirs d'être poussés,

Regards d'être au ciel adressés,

Et puis sanglots, et puis soupirs encore :

En ce même langage Hispal lui repartit :

Tant qu'enfin un baiser suivit :

S'il fut pris ou donné, c'est ce que l'on ignore ${ }^{4 \mathrm{I}}$.

Du coup le narrateur devient un personnage plus réel que les personnages eux-mêmes : tel un montreur de marionnettes, il meut à son gré les fils de la narration en s'ingérant parfois de justifier (hypocritement) la conduite de ses créatures. Comme l'a remarqué Catherine Grisé, il le fait en l'occurrence en se servant de procédés casuistiques ${ }^{42}$. Le langage technique

40. $E C$, p. $67 \mathrm{I}-672$

4I. Ibid., p. 670.

42. Voir C. Grisé, «La casuistique dans les Contes de La Fontaine», Studi francesi, nº 99, 1990, p. 4II-42I. 
de la confession est ici détourné pour «travestir» la réalité jamais dite explicitement du désir sexuel : le narrateur casuiste s'évertue à absoudre chaque nouvelle faute de l'héroïne au nom de ses bonnes intentions, que ce soit "gratitude», "compassion» ou "crainte de pis». Les tours de passe-passe du narrateur, son attitude hypocrite, sa manipulation funambulesque du langage finissent par attirer toute l'attention du lecteur, en la détournant de l'objet de la narration. C'est ainsi que La Fontaine «civilise» la nouvelle italienne, en y infusant une gaieté légère et inoffensive à l'intention du public des salons. L'héroïne n'est plus caractérisée par cette interdiction du langage, qui dans le texte italien en faisait un pur objet sexuel livré à la violence des mâles et aux jeux cruels de la Fortune : au contraire, chaque capitulation sexuelle est précédée par un échange verbal où le séducteur pose ses conditions ou révèle ses désirs et Alaciel accorde ses faveurs en raison de considérations pragmatiques ou morales en apparence inattaquables. Elle devient une raisonneuse, capable de manier subtilement la logique pour justifier son adhésion à une morale "relâchée». Même la succession des assassinats (chaque amant étant tué par son successeur), qui donnait lieu chez Boccace à des scènes d'une violence "shakespearienne», disparaît presque entièrement du conte, puisqu'elle contreviendrait à cette règle de l'unité de ton hautement affirmée dans la préface de $1666^{43}$ : les quelques scènes de violence qui restent sont présentées sur un ton ludique impropre à susciter l'horreur. C'est le cas du corsaire «Grifonio le gigantesque», dont la bestialité grotesque prête à rire non moins que les circonstances de sa mort («on aurait ri de l'aventure») :

Le héros d'un revers coupe en deux l'animal :

Part du tronc tombe en l'eau, disant sa patenôtre,

Et reniant Mahom, Jupin, et Tarvagant,

Avec maint autre dieu non moins extravagant :

Part demeure sur pieds, en la même posture ${ }^{44}$.

Une sorte d'understatement atténue également la scène du carnage des corsaires qui se configure comme un passage indolore du sommeil à la mort :

Presque tout le peuple corsaire

Du sommeil à la mort n'ayant qu'un pas à faire,

Fut assommé sans le sentir ${ }^{45}$.

43. Voir $E C$, p. 605 .

44. Ibid., p. 669.

45. Ibid., p. 678. 
Je crois qu'il faut entendre par «gaieté» précisément ces procédés stylistiques qui caractérisent la voix du narrateur et qui, établissant le primat de la «manière» sur la «matière», délestent de leur poids de réalité les canevas que La Fontaine emprunte à ses modèles.

\section{La voix du fabuliste : Le Loup devenu Berger}

Pour les Fables, la "gaieté» se manifeste essentiellement, on le sait, dans la superposition décalée du monde humain et du monde animal : décalée justement, imparfaite, étant donné l'hybridité plaisante de ces créatures, que La Fontaine a beau jeu de souligner. Le statut de la fable implique un déguisement qui est lui-même souvent thématisé dans les Fables : masque, mauvaise imitation, tentative maladroite pour échapper à son naturel, pour se parer de la peau d'un autre. C'est le cas du Loup devenu Berger, dont la source est encore un auteur italien, $\mathrm{du} \mathrm{XVI}{ }^{\mathrm{e}}$ siècle cette fois : Giovan Mario Verdizzotti. La Fontaine développe certains éléments de parodie déjà présents dans le modèle, mais il les condense, faisant de cette fable l'illustration idéale de notre propos. Il est justement question d'un animal qui se déguise en homme, le contraire de ce qui se passe généralement dans le monde des Fables. Mais la maladresse de son imitation découvre la tromperie et empêche le prédateur d'atteindre son but. "Toujours par quelque endroit Fourbes se laissent prendre» : c'est l'epimithium que le narrateur colle à cet apologue. En même temps cette fable est fondée sur la réactualisation d'une métaphore très répandue dans la réflexion politique à partir du Prince de Machiavel : le prince doit emprunter alternativement la stratégie du renard et du lion, autrement dit la ruse et la force. Appliquée au loup de la fable, l'expression «s'aider de la peau du Renard», c'est donc faire preuve de souplesse, savoir renoncer aux procédés qui nous sont le plus naturels pour recourir à des stratégies alternatives. Mais, prise en mauvaise part, c'est aussi se travestir, essayer de déguiser son naturel, ce qui, dans l'univers des Fables, est le plus banal des leurres : tout déguisement y est promis à l'échec.

Un Loup qui commençait d'avoir petite part

Aux Brebis de son voisinage,

Crut qu'il fallait s'aider de la peau du Renard,

Et faire un nouveau personnage ${ }^{46}$.

46. Le Loup devenu Berger, EC, p. IIо-піг. 
Ces quatre vers évoquent trois personnages de premier plan de l'univers des Fables: le Loup, les Brebis, le Renard. Pourtant ces personnages n'ont pas ici le même statut. Si le Loup et les Brebis, sans considérer la nature allégorique implicite dans le statut des animaux de la fable, ont la consistance de personnages réels, le Renard n'est là que comme image transparente et traditionnelle de la ruse : ce n'est pas un personnage. Il nous ramène à l'univers des hommes. Tout en recouvrant une partie de sa prégnance au contact du monde animal, ce proverbe «s'aider de la peau du Renard" fait tache dans le texte, introduit un élément de "défamiliarisation" qui dénonce la nature sylleptique ${ }^{47}$, donc artificielle, des autres animaux de la fable : il nous dit que le Loup, ainsi que les Brebis, sont en même temps des animaux et des signes. Cette duplicité des animaux de la fable est définitivement dévoilée dans la moralité où, paradoxalement, l'auteur nous ramène du monde des hommes au monde des animaux par un mouvement inverse à celui qui avait déterminé dans le récit un animal à se faire homme. "Quiconque est Loup agisse en Loup" : voici un parfait exemple de syllepse, qui fait écho au proverbe précédent en en inversant la leçon. Les "prédateurs" humains, conscients de leur affinité avec la bête carnassière, doivent se conformer à leur nature profonde sans faux-fuyants hypocrites et maladroits. Un jeu de renvois d'un univers à l'autre s'esquisse donc tout au long de la fable en faisant miroiter l'image du prince machiavélien au cour de la saynète ésopique et le mufle menaçant du loup dans l'application. Rien de tel chez Verdizzotti, où l'image et son référent restaient soigneusement séparés et le basculement de l'une à l'autre se faisait par une transition lourdement didactique : "Tal l'huom bugiardo e di malitia pieno / Rimaner suole [...]» Par cette osmose des deux univers — l'univers signifiant et l'univers signifié —, La Fontaine abolit le partage ésopique entre récit (voile) et moralité (dévoilement). Ces jeux métalinguistiques qui soulignent à loisir le caractère artificiel de la métaphore animalière constituent le legs de l'expérience des Contes dans la fondation d'une fable moderne, destinée à un public mondain. L'ironie couvre le plaisir régressif et irresponsable de la fable permettant au lecteur adulte d'accéder à une source de plaisir qui sans cela lui serait défendue ${ }^{48}$.

47. C'est P. Dandrey qui parle pour les Fables de poétique de la syllepse; voir La Fabrique des Fables, ouvr. cité, p. 215 et suiv.

48. On pourrait peut-être rapprocher cela du désir infantile d'accorder la vie aux choses inanimées dont parle Freud qui en fait dépendre le sentiment de l'Unheimliche chez les adultes. Voir S. Freud, L'Inquiétante Étrangeté et autres essais, trad. de B. Féron, Paris, Gallimard, 1985, p. 55 : "Nous nous souvenons que dès l'âge de ses premiers jeux, l'enfant ne fait généralement pas de distinction nette entre l'animé et l'inanimé, et qu'il éprouve une prédilection particulière à traiter sa 
Mais le jeu de la syllepse se complique du jeu de la parodie, qui demande au lecteur un investissement ultérieur d'intelligence. De même que le Loup se déguise en Berger, la fable se déguise en pastorale. La Houlette, le Hoqueton, la Cornemuse encore une fois ne sont pas de simples objets : ce sont des signes qui évoquent l'univers pastoral. Mais même à ce niveau, le travestissement se solde par une faillite : l'univers pastoral est un univers harmonieux, qui met à distance la violence de l'histoire, tout en la réfléchissant, et qui apaise les tensions et les contrastes par la douceur du chant. La fable est un univers de la violence, régi par les rapports de force entre les êtres, et chaque fois que l'univers pastoral y est évoqué c'est pour dénoncer indirectement le caractère hypocrite et fallacieux de son harmonie ${ }^{49}$. La comédie "pastorale» jouée par le loup, malgré son effort pour afficher tous les signes de son appartenance au monde arcadien ( Il aurait volontiers écrit sur son chapeau...»), est condamnée à l'échec là où la prudence et le soupçon à l'égard des apparences deviennent la condition de la survie.

À la différence du travestissement animal des Fables, le travestissement pastoral, qui s'aggrave de la sublimation du sentiment amoureux que dénoncent les Contes, reste ambivalent et ne se dénonce pas pour tel : il risque de provoquer ces phénomènes de "mimétisme» acritique que dénonçait Sorel dans Le Berger extravagant et que La Fontaine lui-même dénoncera dans $P$ sychés $e^{\circ}$. Par rapport à la mystification pastorale, dans la fable paradoxalement la réalité reprend ses droits : c'est par le dévoilement des lois impitoyables qui régissent aussi bien la nature que la société que la fable prétend dire un mot de vérité sur l'univers des hommes. Derrière la fiction avouée et soulignée à loisir s'esquisse donc dans les Fables un mouvement inverse à celui des Contes: là, à partir d'une représentation sans complaisance du plaisir sexuel, on aboutissait à l'évidement de toute réalité, de toute corporalité, sous l'action corrosive des jeux intertextuels et métalinguistiques, ici sous la fiction enfantine allégrement dénoncée, sous l'écorce usée des apologues ésopiques perce le pouvoir de démystification

poupée comme un être vivant. [...] La source du sentiment d'inquiétante étrangeté ne serait donc pas ici une angoisse infantile, mais un désir ou même simplement une croyance infantile.»

49. Dans la fable-programme Contre ceux qui ont le goût difficile, le mode pastoral, identifié par ses éléments les plus conventionnels et par une fadeur qui le disqualifie, est convoqué à côté de l'épique pour définir par contraste le mode de la fable, le plus approprié à l'esprit du poète. Pour le rapport entre La Fontaine et la tradition pastorale, voir J.-P. Collinet, «La Fontaine et la Pastorale», dans La Fontaine et quelques autres, Genève, Droz, 1992, p. I29-I42.

50. Notamment dans le passage relatif à la «glucomorie» de Psyché, qui «parlait étant seule, ainsi qu'en usent les amants / dans les vers et dans les romans» (voir CED, p. I55). 
que, dans la cadre d'une nouvelle anthropologie, la métaphore animale prétend exercer dans la seconde moitié du XVII ${ }^{\mathrm{e}}$ siècle contre la célébration humaniste de la dignitas hominis ${ }^{\text {s. }}$.

Ces analyses de texte nous ont donc amené à dégager une opposition nette entre les Contes et les Fables que l'étiquette commune de gaieté semblait assimiler entièrement. Il y a bien chez La Fontaine une volonté de rapprocher les deux genres, qui s'apparentent à ses yeux surtout par leur attitude désinvolte à l'égard du critère de la vraisemblance, de plus en plus important pour la littérature du Grand Siècle : de même que l'histoire de Psyché, ce sont des «badineries", des «bagatelles», qui permettent néanmoins, par leur abandon contrôlé à la fiction, par l'ingéniosité de leur gaieté lucide de réactiver des sources de plaisir inaccessibles à l'homme du monde. Pourtant, dans les Contes, les allusions plaisantes aux sources et l'éloquence débordante et funambulesque du narrateur finissent par déterminer un primat absolu de la "manière» sur la «matière», qui réduit l'écriture littéraire à un jeu sans conséquence qui a perdu toute prise sur le réel. L'«idéologie» qui informe l'œuvre, très proche de celle des comédies de Molière (légitimité du désir naturel, condamnation de la jalousie et de l'amour possessif, réprobation des mariages forcés, dénonciation de l'hypocrisie des moines et des dévots ${ }^{52}$ ), perd toute sa portée critique par l'outrance paradoxale avec laquelle le narrateur la fait valoir ${ }^{33}$. Dans les Fables, au contraire, la naïveté apparente permet de mieux faire ressortir la valeur critique de l'allégorie animale dans la culture du Grand Siècle. L'apologue ésopique est obligé de par son statut à représenter les conflits dans une forme hyperbolique et condensée : hyperbolique parce que le conflit se radicalise en lutte pour la survie et se termine presque toujours par l'élimination physique voire la dévoration du plus faible ou du moins rusé, condensée parce que la brièveté requise par les lois du genre en resserrant l'espace diégétique ajoute à la violence et à la soudaineté du dénouement. Aucun autre genre ne se prêtait aussi bien à décrire un monde désenchanté, machiavélien, dominé par la force et par la ruse, où la noblesse de la nature humaine est de plus en plus défigurée par des pulsions animales. La fable entre les mains de La Fontaine devient un outil acéré pour déchirer tout voile mystifiant et toute représentation idéalisée de la vie des hommes en société, y compris celle que proposent

5I. Voir M. Fumaroli, Introduction à Jean de La Fontaine, Fables, Paris, Imprimerie nationale, "La Pochothèque», I985, p. Xxxiv et suiv.

52. Voir P. Bénichou, Morales du Grand Siècle, Paris, Gallimard, coll. "Folio", I948, p. 210-296.

53. Qu'on songe à l'ingénieuse apologie du cocuage qui ouvre le conte de La Coupe enchantée. 
d'autres genres littéraires, comme la pastorale. La "gaieté» a la fonction à la fois de «faire passer» et de compenser par l'euphorie narrative une image de l'homme qui est presque intolérable à force de pessimisme et qui ressemble de près à celle des Maximes de La Rochefoucauld ou des Pensées de Pascal. C'est donc un double plaisir que les Fables nous offrent : aux plaisirs de la "gaieté», de cette fiction enfantine et régressive rehaussée par les jeux de la syllepse, s'associe le plaisir «mélancolique» de lever le voile avec le fabuliste sur l'homme tel qu'il est. 